\title{
BROOKHTWEN
}

NATIONAL LABORATORY

BNL-91049-2010-CP

\section{Residual-gas-ionization beam profile monitors in $\mathrm{RHIC}$}

\author{
R. Connolly, J. Fite, S. Jao, S. Tepikian, C. Trabocchi
}

Presented at the 2010 Beam Instrumentation Workshop (BIW10)

Santa Fe, New Mexico

May 2-6, 2010

\author{
Collider-Accelerator Department \\ Brookhaven National Laboratory \\ P.O. Box 5000 \\ Upton, NY 11973-5000 \\ www.bnl.gov
}

\begin{abstract}
Notice: This manuscript has been authored by employees of Brookhaven Science Associates, LLC under Contract No. DE-AC02-98CH10886 with the U.S. Department of Energy. The publisher by accepting the manuscript for publication acknowledges that the United States Government retains a non-exclusive, paid-up, irrevocable, world-wide license to publish or reproduce the published form of this manuscript, or allow others to do so, for United States Government purposes.
\end{abstract}

This preprint is intended for publication in a journal or proceedings. Since changes may be made before publication, it may not be cited or reproduced without the author's permission. 


\section{DISCLAIMER}

This report was prepared as an account of work sponsored by an agency of the United States Government. Neither the United States Government nor any agency thereof, nor any of their employees, nor any of their contractors, subcontractors, or their employees, makes any warranty, express or implied, or assumes any legal liability or responsibility for the accuracy, completeness, or any third party's use or the results of such use of any information, apparatus, product, or process disclosed, or represents that its use would not infringe privately owned rights. Reference herein to any specific commercial product, process, or service by trade name, trademark, manufacturer, or otherwise, does not necessarily constitute or imply its endorsement, recommendation, or favoring by the United States Government or any agency thereof or its contractors or subcontractors. The views and opinions of authors expressed herein do not necessarily state or reflect those of the United States Government or any agency thereof. 


\title{
RESIDUAL-GAS-IONIZATION BEAM PROFILE MONITORS IN RHIC*
}

\author{
R. Connolly, J. Fite, S. Jao, S. Tepikian and C. Trabocchi \\ Brookhaven National Lab, Upton, NY, USA
}

\section{Abstract}

Four ionization profile monitors (IPMs) in RHIC measure vertical and horizontal beam profiles in the two rings (yellow and blue). These work by measuring the distribution of electrons produced by beam ionization of residual gas. In 2007 a prototype of a new design was installed in the yellow ring. During the 2007-2008 run it proved to be almost completely free from backgrounds from rf coupling, electron clouds and $\mathrm{x}$-rays from upstream beam loss. In 2009 four new IPMs were built based on the prototype. During the 2009 shutdown two of these IPMs were installed. This paper describes the new IPMs and shows data from the 2010 beam run. The new IPMs have been extremely important in the commissioning of the RHIC stochastic cooling system.

\section{INTRODUCTION}

The Relativistic Heavy-Ion Collider (RHIC) at Brookhaven National Laboratory is a pair of concentric synchrotrons in which counter-rotating beams intersect at six points [1]. Beams ranging in mass from protons $\left(E_{\max }=250 \mathrm{GeV}\right)$ to fully-stripped gold $\left(E_{\max }=100\right.$ $\mathrm{GeV} /$ nucleon) are accelerated and stored for several hours. There are detectors at two of the six intersection points for physics experiments with colliding beams.

Transverse beam profiles in RHIC are measured with ionization profile monitors (IPMs)[2,3,4,5]. An IPM collects the electrons in the beamline resulting from residual gas ionization during a bunch passage. The electrons are swept transversely from the beamline and collected on 64 strip anodes oriented parallel to the beam axis. After each bunch passes through the detector, the charge pulses are amplified, integrated, and digitized to give the bunch profile. Four IPMs are installed in RHIC to measure vertical and horizontal profiles in the two rings. Similar detectors are used at Fermi National Lab [6], DESY [7], and CERN [8].

The beam profile is measured primarily to find the transverse beam emittance which is the area occupied by the beam in transverse phase space, ie. $\left(x, p_{x}\right)$ [3]. The beam radius at the IPM is equal to the square root of the betatron function, $\beta(\mathrm{s})$, times the emittance where $\mathrm{s}$ is the azimuthal coordinate of the IPM. The value of $\beta(\mathrm{s})$ is either calculated or measured and the beam radius is determined by profile measurements.

Work performed by employees of Brookhaven Science Associates, LLC under Contract No. DE-AC02-98CH10886 with the U.S. Department of Energy

\section{DETECTOR}

Figure 1 is a schematic of the detector and its associated electronics in the accelerator tunnel. Figure 2 is a cutaway drawing and fig. 3 is a photo of the transducer. The detector chamber is located in a section of $10 \times 15 \mathrm{~cm}$ rectangular pipe. In an $18-\mathrm{cm}$-long section of this beam pipe half of the pipe has been removed and a saddle piece inserted. This saddle piece is biased at $-6 \mathrm{kV}$ producing an accurate transverse electric field of $6 \times 10^{4} \mathrm{~V} /$ $\mathrm{m}$.

A screen-covered rectangular opening in the grounded half of the beam pipe allows the signal electrons to pass through the image-current path to the detection assembly. The detection assembly is a $8 \times 10 \mathrm{~cm}$ microchannel plate (MCP) with a 64-channel anode etched on a ceramic circuit board. The collector board with attached MCP is located in an rf enclosure to prevent coupling to the beam. The only opening to this enclosure, which the signal electrons pass through, is a grounded aluminum honeycomb which attenuates rf by $80 \mathrm{~dB}$. Between the image-current bypass screen and the rf honeycomb window is a wire-screen control grid for gating the signal electrons off between measurements.

Each channel is connected via vacuum feedthrough to a preamplifier which drives a shielded twisted-pair transmission line to a 10MSPS VME digitizer.

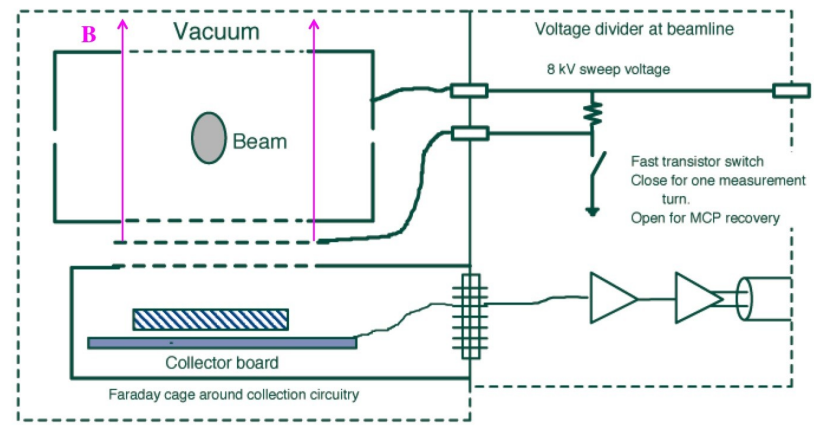

Figure 1: Schematic of detector and electronics located at the beamline.

In routine operation the signal-gating grid is normally biased at the sweep voltage of $-6 \mathrm{kV}$ which prevents the signal electrons from passing. It is pulled to ground for a single turn $(12 \mu \mathrm{s})$ for measurement and returned to the off voltage. During this turn the digitizers are triggered. Each channel of an MCP has a dead time of about $1 \mathrm{~ms}$ after firing. The intense heavy-ion beams of RHIC create 
such a large signal flux that the MCP becomes charge depleted very quickly. After a single measurement turn the grid is returned to $-6 \mathrm{kV}$ for about 100 machine revolution periods for the MCP to recover. This is repeated 100 times and the data are averaged.

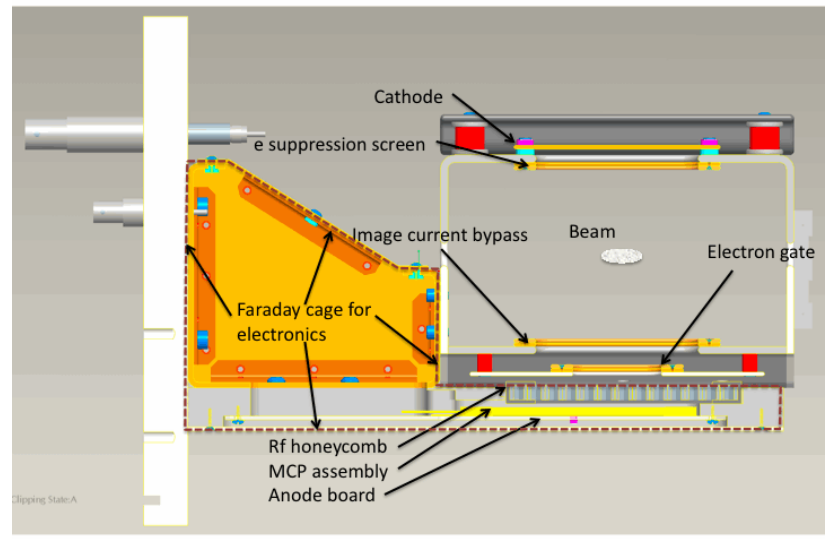

Figure 2. Cutaway drawing of the IPM. The HV sweep electrode is on top and MCP and anode board on bottom.

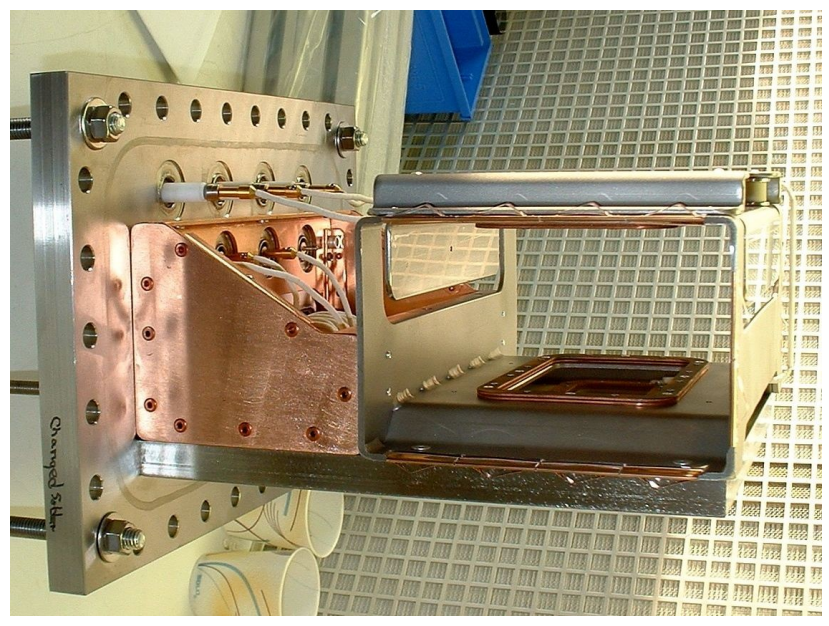

Figure 3. Assembled IPM transducer.

\section{BEAM MEASUREMENTS}

In 2009-10 RHIC ran gold beams and the IPMs were set up for single-bunch measurements. In this mode the digitizers can be triggered on any of the buckets desired. During most of the beam run we monitored the bunch in bucket 121. This bucket has beam in all fill patterns and is not affected by the tune measurements or gap cleaning. Figure 4 is the display page of the IPM application showing the data, Gaussian fits and calculated widths and emittances.

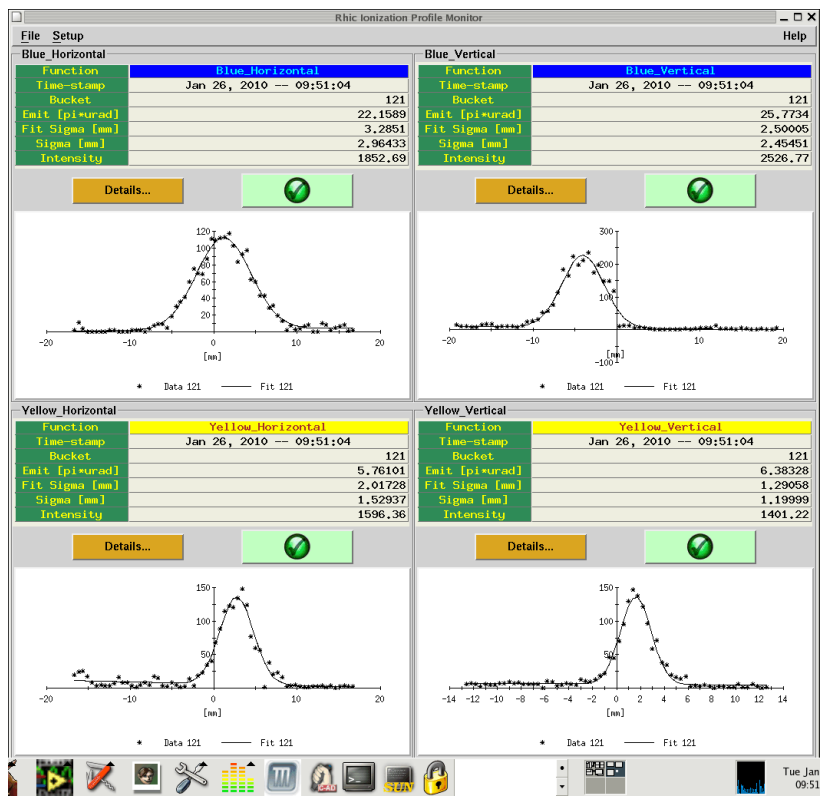

Figure 4. The IPM display page.

The most interesting development of this beam run was the commissioning of the RHIC stochastic cooling system $[9,10]$. The yellow-ring system was the first to operate and ran more consistently than the blue ring system. Figure 5 is a four-hour emittance record from the IPMs showing the effect the cooling system had on the beam in the yellow ring. Yellow cooling is turned on shortly after 18:20 and reduces the emittances in both planes of yellow from $(13 \pi) \mu \mathrm{m}$ to $(5 \pi) \mu \mathrm{m}$ in about an hour. Emittances in blue continue to grow during this time. The profiles shown in fig. 4 were taken with yellow cooling on and show dramatically smaller beams in the yellow ring. Figure 6 is the log entry from the first time cooling was done in the blue ring. At 17:30 yellow cooling was turned off and at 18:00 blue cooling is turned on. 


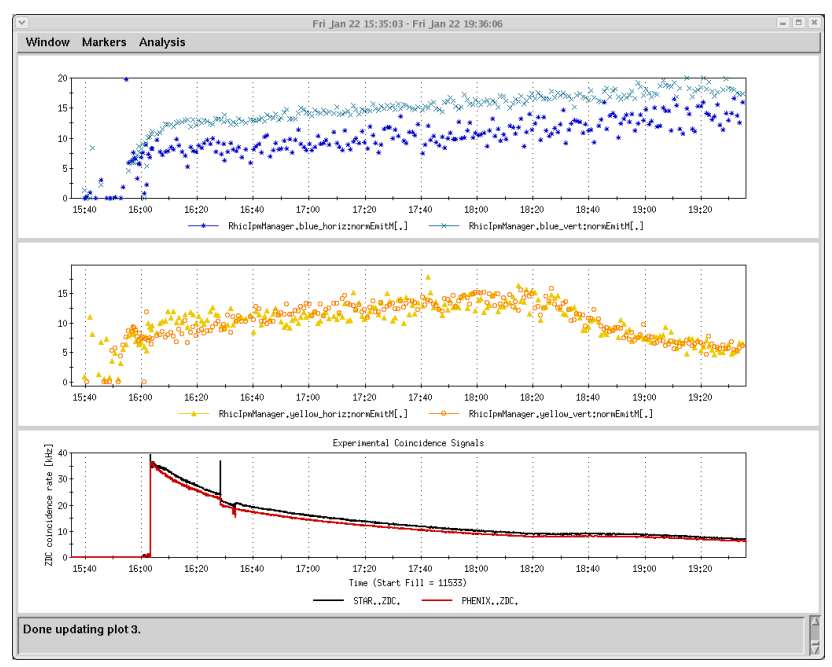

Figure 5. IPM record showing yellow-ring cooling.

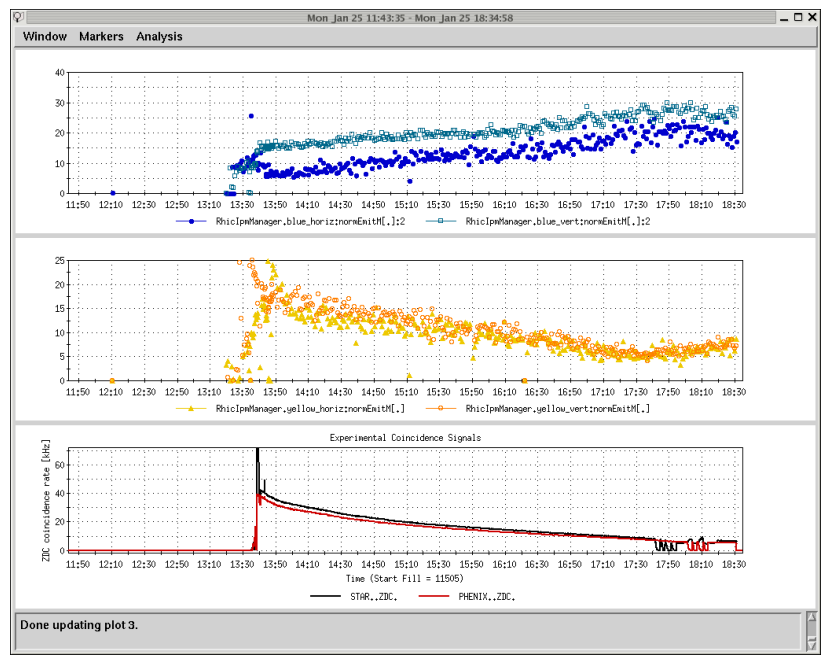

Figure 6. IPM record of first blue-ring cooling.

The IPMs are turned on at each machine fill. They take measurements at 30 s intervals during the acceleration ramp and at 1 minute intervals during the store. All beam and system parameters are recorded at each measurement. Figure 7 is a log entry showing the IPM records over a weekend. The top plot shows the IPM data and the bottom plot is the beam current record. During this weekend the blue-ring emittances were nearly constant but the yellow emittances fluctuated over a range of $(20 \pi) \mu \mathrm{m}$ to $(40 \pi) \mu \mathrm{m}$.

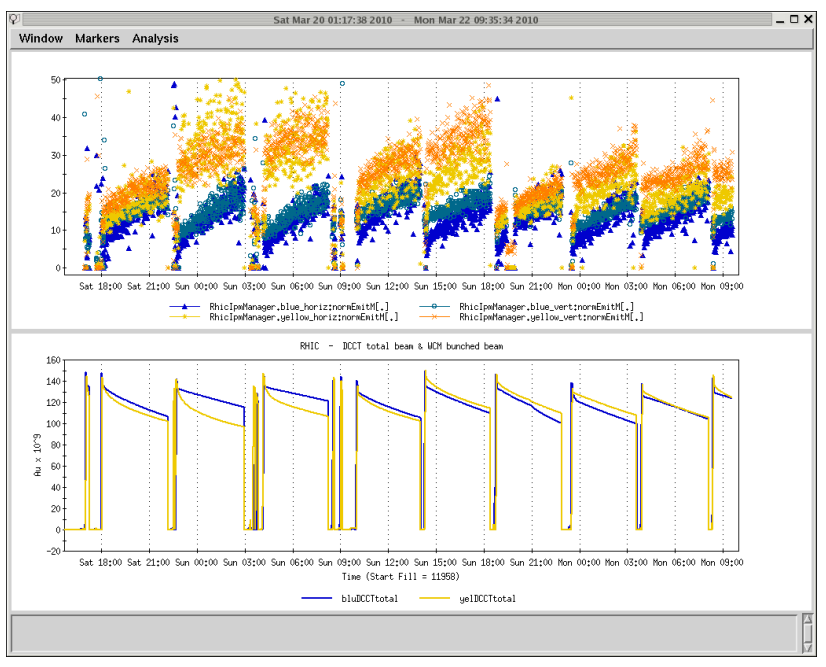

Figure 7. IPM emittance records over a weekend.

\section{DISCUSSION}

The last paper on the RHIC IPMs, presented at the 2005 PAC [2], reported issues with the electron-control grid coupling to the beam and rf noise from the beam coupling into the detection electronics. Since that paper was written we have moved the control grid outside of the beam image-current path and placed the MCP and collector inside an rf enclosure inside the vacuum chamber. These improvements have almost completely eliminated backgrounds.

Also since the last paper, emittance measurements have been implemented on RHIC using a movable Schottky cavity [11]. A resonant cavity is moved transversely on the beam line. At each position the ratio of the power in the revolution line to the sum of the powers in the two betatron sidebands is measured. A parabola is fitted to the data to find the rms beam size, $\sigma$, and the dispersion offset. These measurements agree exactly with the IPMs.

\section{ACKNOWLEDGEMENTS}

The prototype of the new IPM was built by Dave Kipp. Tom Russo developed the procedure for soldering the signal wires to the ceramic circuit boards.

\section{REFERENCES}

[1] http://www.rhic.bnl.gov/

[2] R. Connolly, R. Michnoff and S. Tepikian, "Residual-gasionization Beam Profile Monitors in RHIC," Proc. 2005 PAC, Knoxville, TN.

[3] R. Connolly, R. Michnoff, T. Moore, T. Shea, and S. Tepikian, Nucl. Instr. and Meth. A 443 (2000) 215-222. 
[4] Connolly, R., Cameron, P., Michnoff, R., and Tepikian, S., Performance of the RHIC IPM, Proc. 2001 PAC, Chicago.

[5] R. Connolly, M. Grau, R. Michnoff and S. Tepikian, "The IPM as a Halo Measurement and Prevention Diagnostic," Halo '03, ICFA Advanced Beam Dynamic Workshop, Montauk, NY, USA (2003).

[6] J. R. Zagel, D. J. Harding, B. C. Brown, H. D. Glass, L. R. Kozien, S. M. Pruss, J. T. Volk, "Permanent Magnet Ion Profile Monitor at the Fermilab Main Injector." TPAH036, Proc. 2001 PAC., Chicago.

[7] K. Wittenburg, "Experience with the Residual Gas Ionization Beam Profile Monitors at the DESY Proton Accelerators," Proc. 1992 EPAC., 24.3.-28.3, Berlin, Germany and DESY HERA 92-12
[8] C. Fischer, "Results on Prototype Measurements for LHC Beam Instruments," Proc. 2001 DIPAC, Grenoble, France.

[9] M. Blaskiewicz, J.M. Brennan and F. Severino, Phys. Rev. Let. 100, 174802 (2008).

[10] J.M. Brennan and M. Blaskiewicz, "Stochastic Cooling in RHIC," Proc. 2009 PAC, Vanouver, BC, Canada.

[11] K.A. Brown, M. Blaskiewicz, C. Degen and A. Della Penna, Phys. Rev. Spec. Topics-Accel. and Beams, 12, 012801 (2009). 